\title{
Long Term Progression Free Survival Achieved by Maintenance "Second-Line" Polychemotherapy with Gemcitabine/Paclitaxel in Metastatic Urothelial Cancer of the Renal Pelvis-A Case Report
}

\author{
Andreas Bannowsky ${ }^{1}$, Hermann Van Ahlen ${ }^{1}$, Klaus-Peter Jünemann ${ }^{2}$ \\ ${ }^{1}$ Department of Urology, Klinikum Osnabrück, Germany; ${ }^{2}$ Department of Urology, University Hospital Schleswig-Holstein, Campus \\ Kiel, Germany. \\ Email:andreas.bannowsky@klinikum-os.de
}

Received June 21 ${ }^{\text {st }}$, 2010; revised July $5^{\text {th }}$, 2010; accepted July $22^{\text {nd }}, 2010$.

\begin{abstract}
Moderate activity of systemic chemotherapy for advanced urothelial cancer has been reported for more than 30 years. Only with the advent of potent combination therapy clinically significant response rates as well as prolonged survival were documented. The therapeutic effect of a "second-line" polychemotherapy in metastatic upper tract urothelial cancer is largely unknown caused by the small number of cases and poor prognosis. We report an interesting case of a 59-year-old man suffering from urothelial cancer of the renal pelvis with pulmonary, lymphogenic and bone metastases who showed an unexpected response to a "second-line"chemotherapy after only 2 treatment cycles of Gemcitabine/Paclitaxel (partial remission) after 24 treatment cycles Gemcitabine / Cisplatin in "stable disease" and progression between the treatment intervals. We performed maintenance "second-line" therapy for 24 cycles and the patient showed a remarkable persisting response 54 months after operation.
\end{abstract}

Keywords: Chemotherapy, Renal Pelvis, Urothelial Cancer, Paclitaxel

\section{Introduction}

The gold standard treatment for advanced urothelial cancer of the renal pelvis is radical nephroureterectomy with the complete removal of a bladder cuff around the ipsilateral ureteral orifice. In case of metastatic disease a cisplatin based polychemotherapy follows as an adjuvant or palliative treatment. These, often nephrotoxic chemotherapy can lead to long term remission or permanent cure in only a small number of patients. We report the remarkable progression of a patient with a metastasized urothelial cancer of the renal pelvis in the time before vinflunine had become available for second-line treatment.

\section{Case Report}

A tumor nephrectomy on the right side was performed on a 59 year old patient after suspicion of a pulmonary, lymphogenic and bone metastasized kidney cancer (suggested RCC). The pathology indicated an advanced urothelial cancer of the renal pelvis (tumor stage pT3a). The primary staging computertomography (CT) showed extensive metastatic disease with involvement of the retroperitoneal lymph nodes, pulmonary metastases and bone metastatic disease in the right Os ileum. After palliative analgetic and stabilizising radiation (30 Gy) of the right Os ileum, six cycles of polychemotherapy with Gemcitabine $\left(1200 \mathrm{mg} / \mathrm{m}^{2}\right)$ and Cisplatin $\left(70 \mathrm{mg} / \mathrm{m}^{2}\right)$ were performed. At the first re-staging a "stable disease" (SD) was shown by computertomography. During treament, we again performed six cycles of polychemotherapy with Gemcitabine/Cisplatin, due to progressive disease. After this treatment, SD for five months occurred, followed by another progression. In total the patient received 24 cycles of polychemotherapy with Gemcitabine/Cisplatin because of recurrent progressive disease 
between the therapeutic intervals.

After these 24 cycles the patient developed a significant increase of the disseminated pulmonary and retroperitoneal metastases in only six weeks. Therefore a "second-line" polychemotherapy with Gemcitabine $\left(1000 \mathrm{mg} / \mathrm{m}^{2}\right)$ and Paclitaxel $\left(175 \mathrm{mg} / \mathrm{m}^{2}\right)$ was initiated. After 2 cycles of Gemcitabine/Paclitaxel a significant reduction in size and number of the pulmonary metastases (Figure 1 and 2) and of the retroperitoneal lymph nodes was observed. Since then we performed this polychemotherapy as a sequencially applied maintenance therapy in a 21-day cycle. At the moment the patient lives without complaints and has a permanent partial remission (PR) with significant size reduction of the pulmonary metastases (Figure 3) after 24 cycles of Gemcitabine/ Paclitaxel. He reports a significantly increased physical condition in comparison to the Cisplatin-based chemotherapy. Since the initiation of the therapy with Paclitaxel and Gemcitabine, no anaemia (WHO grade 3 or 4) or febrile neutropenia (WHO grade 3 or 4 ) occurred. In spite of the extensive amount of nephrotoxic chemotherapy the kidney function is still satisfactory with a creatinine clearance of $70 \mathrm{ml} / \mathrm{min}$.

\section{Discussion}

Urothelial carcinoma of the kidney is relatively an uncommon tumor and occurs in about $10 \%$ of all malignant tumors of the kidney. Regarding all urothelial cancers of the urinary tract, the renal pelvis cancer occurs in about $5 \%$ [1]. $40-70 \%$ of the tumors, like the urothelial cancer of the bladder, shows an association with tobacco smoking. The main symptom is the painless haematuria, followed by flank pain caused by obstruction due to ureteral tumor manifestation. The correct imaging is per-

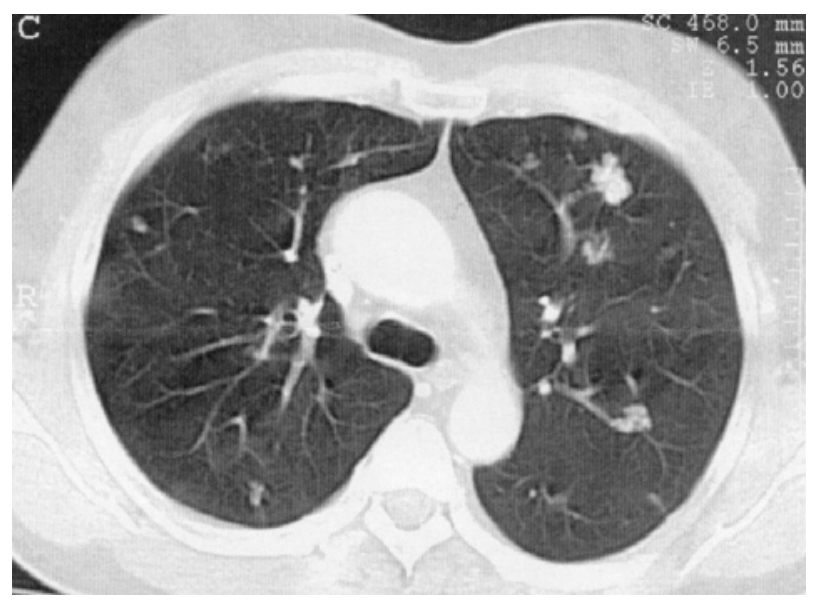

Figure 1. CT of the chest: disseminated pulmonary metastatic disease after 24 cycles Gemcitabine/Cisplatin prior to "second-line" therapy.

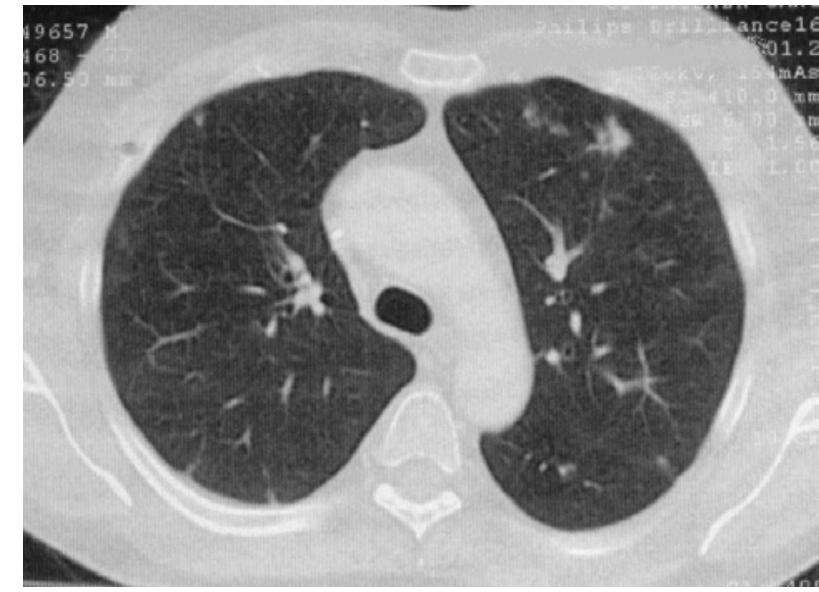

Figure 2. CT of the chest: regression of the pulmonary metastases after 2 cycles Gemcitabine/Paclitaxel, partial remission.

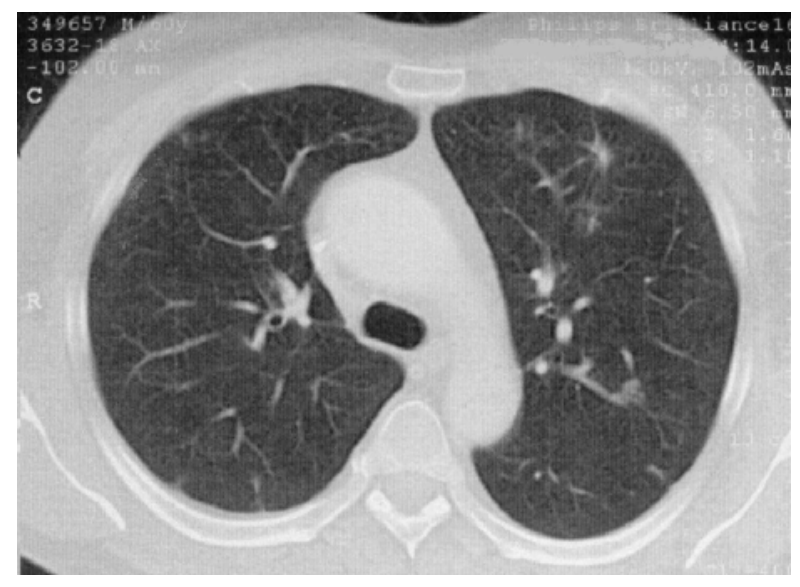

Figure 3. CT of the chest: regression of the pulmonary metastases after 24 cycles Gemcitabine/Paclitaxel, persisting partial remission.

formed through intravenous urography (IVU), CT scan, retrograde ureteropyelography and endoscopy with biopsy.

The standard therapy of the renal pelvis cancer is the nephroureterectomy with bladder cuff excision and regional lymph node dissection. Following nephrectomy, tumor relapse rates in the ureter stump are noticed in up to $23 \%$ of the patients. The organ sparing procedures (open, ureterorenoscopic and percutaneous tumor excision) are currently under investigation.

Depending on the tumor stage the 5-year survival rate after nephroureterectomy is $90-95 \%$ for pTa and pT1, $85 \%$ for pT2-/3- and 38\% for pT4- tumors. Due to small numbers, the impact of the adjuvant chemotherapy is debated. No sufficient data are available due to small number of patients and poor survival rates to make firm statements about survival expectancy. The 5-year sur- 
vival rate of patients with lymphogenic or haematogenic metastasized urothelial cancer is only $15-20 \%$ [2]. The median survival with a metastatic upper urinary tract cancer with visceral metastasis (pulmonary/liver/bone) is about 10 months and can be increased to $14-15$ months with a polychemotherapy with a combination of Gemcitabine and Cisplatin or the "gold standard" chemotherapy with MVAC (methotrexate, vinblastine, adriamycine and cisplatin) with a total response rate of $49 \%$ for Gemcitabine/Cisplatin and 46\% with MVAC-treated patients $[3,4]$. The most common toxicities in both therapy schemes are the haematological toxicities with grade III/IV-anaemia (18-27\%), thrombopenia (21-57\%) as well as grade III/IV-neutropenia (71-82\%). Due to the proven advantages in the toxicity profile with most likely the same effectiveness, Gemcitabine/Cisplatin has become the standard treatment [4]. Other combinations with Paclitaxel and Carboplatine show an increase of a 5 -year survival rate up to $52 \%$ for the lymphogenic metastasized urothelial cancer [5]. In case of further disease progression, no data was available at the moment of progression in this patient with regards to an effective "second-line" therapy of this tumor entity. Vinflunine is a new promising "second-line" strategy after first-line cisplatin-containing regimen for metastatic bladder cancer [6], and is considered as an option for "third-line" treatment for this particular patient.

In agreement with the therapy protocol of the study: AUO-Nr. AB20/99 (randomized phase III study for "second-line" therapy of the cisplatin pre-treated metastasized urothelial cancer with Gemcitabine/Paclitaxel), we successfully performed the therapy with Gemcitabine and Paclitaxel as a sequencially maintenance therapy. Including the patient in this particular study was not possible at that time, due to the formerly exclusion criteria of a prior gemcitabine-based therapy.

Despite initially extreme unfavourable prognosis and excessive cumulative dosage of chemotherapy, in this case the patient lives 54 months after operation in overall good condition (Karnofsky-performance status 100\%) after a total of 24 cycles of Gemcitabine/ Cisplatin and 24 cycles of Gemcitabine/Paclitaxel and is in partial remission.

This patient had an unexpected and remarkable long-term process with persisting response to a "second-line" chemotherapy with Gemcitabine/Paclitaxel. Especially in treating younger patients, this interesting case impressively shows the need for using newer polychemotherapy schemes as a therapy, despite obvious therapy failure of a well-established chemotherapy and therefore being able to offer the patient an additional therapeutic option. The authors would like to point out, to include suitable patients in controlled multicentre studies to guarantee the collection of data and therefore lay a foundation of further therapy recommendations.

\section{REFERENCES}

[1] S. Hauser and U. E. Studer, "Therapy of the Renal Pelvis Carcinoma,” Urologe, Vol. 40, No. 6, 2001, pp. 452-455.

[2] J. J. Munoz, L. M. Ellison, "Upper Tract Urothelial Neoplasms: Incidence and Survival during the Last 2 Decades,” Journal of Urology, Vol. 164, No. 5, 2000, pp. 1523-1525.

[3] C. N. Sternberg, "Metastatic Bladder Cancer: Role of Chemotherapy and New Agents," EAU Update Series, Vol. 1, No. 2, 2003, pp. 108-117.

[4] J. Lehmann, M. Retz, M. Hack, S. Siemer and M. Stöckle, "Systemic Chemotherapy of Urothelial Cancer," Onkologie, Vol. 26, Sup. 4, 2003, pp. 18-25.

[5] A. Bamias, C. Deliveliotis, G. Fountzilas, et al., “Adjuvant Chemotherapy with Paclitaxel and Carboplatin in Patients with Advanced Carcinoma of the Upper Urinary Tract: a study by the Hellenic Cooperative Oncology Group,” Journal of Clinical Oncology, Vol. 22, No. 11, 2004, pp. 2150-2154.

[6] S. Culine, C. Theodore, M. De Santis, et al., "A phase II study of Vinflunine in Bladder Cancer Patients Progressing after First-line Platinum-containing Regimen,” British Journal of Cancer, Vol. 94, 2006, pp. 1395-1401. 